\title{
Effects of Shear Connectors on Plate-reinforced Composite Coupling Beams of Short and Medium-long Spans
}

\author{
R.K.L. SU ${ }^{1 *}$, H.J. PAM ${ }^{2}$ and W.Y. LAM ${ }^{3}$
}

ABSTRACT: Experimental studies on the newly proposed design of plate-reinforced composite (PRC) coupling beams have been carried out. Previous results have demonstrated the useful application of this design in coupling beams of medium spandepth ratios $(l / h)$ under both inelastic seismic and elastic wind loading. This paper presents further experimental studies on five PRC coupling beams which investigated the importance of shear connectors on the plate/reinforced concrete composite action. Three medium-length $(l / h=2.5)$ and two short $(l / h=1.17)$ PRC coupling beams, each containing a vertically embedded steel plate, were tested under reversed cyclic loading. While one short beam was welded with expanded metal meshes on the plate surfaces, others were welded with shear studs on the plates in the wall regions and/or the beam spans. Results showed that the expanded metal meshes could not work effectively, and while the shear studs in the beam span would only slightly increase the beam capacity, those in the wall regions would contribute much to improve the inelastic beam performance.

Key Words: Coupling beam, composite, shear stud, steel plate, seismic design

1 Assistant Professor, Dept. of Civil Engineering, The University of Hong Kong, Pokfulam Road, Hong Kong

Tel. +85228592648

Fax. +85225595337

E-mail: klsu@hkucc.hku.hk

*Corresponding Author

2 Associate Professor, Dept. of Civil Engineering, The University of Hong Kong, 
Pokfulam Road, Hong Kong

${ }^{3}$ Ph.D. Student, Dept. of Civil Engineering, The University of Hong Kong, Pokfulam Road, Hong Kong

\section{INTRODUCTION}

\section{Research Background}

Reinforced concrete (RC) coupling beams spanning across openings in wall piers are often the most critical elements in a coupled shear wall structure. These beams are employed to transfer loading between wall piers for a coupling action in resisting lateral loading. Due to their small sectional dimensions, the stiffness of these beams is low compared with the wall piers. As a result, they often experience very high induced bending and shear stresses under lateral loads.

In order to prevent fatigue failures under wind-induced cyclic loading, coupling beams have to remain elastic under service conditions. Conventional RC coupling beams with longitudinal flexural reinforcement and vertical shear reinforcement are thus made deep when large initial stiffness and shear capacities are required. However, conventional RC coupling beams with small span-depth ratios $(l / h<1.3$ to 1.4$)$ are prone to brittle failure in the form of diagonal shear or sliding cracking under earthquake-induced inelastic reversed cyclic loading [1,2].

In order to ensure a sufficient shear capacity under large wind loading, especially in high-rise buildings, and to insure survival of the structure under high intensity cyclic loading during an earthquake, alternative designs are required for improving the strength, stiffness, ductility, and energy dissipation abilities of coupling beams. To cater for this need, various alternatives have been proposed. These include diagonally reinforced concrete coupling beams [3], RC coupling beams with rhombic reinforcement layouts [4], RC beams with plate reinforcement [5], steel I-beams [6], composite beams with structural steel beams embedded in nominally reinforced concrete [7] and concrete filled 
steel tubes [8]. The research on PRC coupling beams was also conducted by the authors [9] with the aim of providing the construction industry with a feasible alternative design.

\section{Characteristics of PRC Coupling Beams}

Figure 1 shows the reinforcement cages of two PRC coupling beams on site with medium and small span-depth ratios respectively. The one with the medium span-depth ratio on the left was the first application of the PRC coupling beam design proposed by the co-author Dr. Su in a private development project in Hong Kong. In this design, a steel plate is vertically embedded into the conventionally reinforced beam section across the whole span. Throughout the span, shear studs are welded on both vertical faces of the plate along the top and the bottom longitudinal reinforcement to enhance the plate/RC composite action. The plate is anchored in the wall piers and shear studs are provided in these regions to increase the plate bearing strength.

Sliding cracking is a major problem causing brittle failure in a conventional $\mathrm{RC}$ coupling beam. This occurs when vertical cracks are formed at the beam-wall interfaces under load reversals. As the shear transfer across the beam-wall interfaces through aggregate interlock is inactivated by the cracks, the coupling beam will slide against its adjacent wall piers and fail in a brittle manner [10]. With the embedded steel plate of a PRC coupling beam framing into the wall piers, a continuous shear transfer medium far less affected by concrete cracking at the beam-wall interfaces during the inelastic stage is provided, thus preventing brittle failure and increasing the rotational ductility of the beam.

As the provision of steel plates also increases the initial stiffness and the strength of a coupling beam, smaller beam sizes can be allowed to cater for architectural requirements. 


\section{Experimental Investigation on the Performance of PRC Coupling Beams}

A total of eight medium-length coupling beam specimens $(l / h=2.5)$, including two conventional RC coupling beams and six PRC coupling beams, and two short PRC coupling beam specimens $(l / h=1.17)$ have been tested under reversed cyclic loading in the experimental investigation conducted by the authors. Some of the experimental results on the medium-length coupling beams have been presented in previous publications [11-16]. The results demonstrated the prevailing performance of PRC coupling beams over conventional RC coupling beams under both elastic loading and inelastic imposed deformations. By embedding a steel plate of thickness about one tenth of the beam width into an RC coupling beam with conventional reinforcement, the strength was almost doubled and the total energy dissipated almost tripled [16]. The importance of shear studs, especially in the wall anchorage regions of the plate, on the seismic performance of a PRC coupling beam has also been recognized [12,14].

This paper discusses further the role of shear studs in both medium-length and short PRC coupling beams, as well as the possibility of using expanded metal meshes as an alternative plate/RC shear transfer medium in PRC coupling beams. The preliminary plate anchorage design procedure proposed previously [16] is also revised based on the experimental observations on medium-length PRC coupling beam specimens.

\section{EXPERIMENTAL PROGRAM}

\section{Test Specimens}

Previous experimental results of medium-length PRC coupling beams $(l / h=2.5)$ showed that the seismic performance of a PRC coupling beam would be seriously affected by the absence of shear studs on the embedded steel plate [12,14]. In order to investigate the contributions of shear studs in the beam span and the wall anchorage regions, two more medium-length PRC coupling beams were fabricated. Unit CB 
contained shear studs in the beam span and Unit CW in the wall regions (Figure 2). Their performances will be compared with those of a previously tested coupling beam $[12,14]$, which contained a full set of shear studs (Unit CF) as described in the above section.

With the applicability of the proposed design verified by the experimental results of the medium-length PRC coupling beams, the investigation was extended to short coupling beams, which are more critical than medium or long coupling beams under inelastic seismic deformations. Two geometrically identical specimens of $l / h=1.17$ were fabricated, one with expanded metal meshes welded on the plate surfaces (Unit SF) and the other with stud shear connectors on the plate (Unit BS). The two coupling beams were fabricated according to two different approaches in the plate anchorage design. Unit SF was designed with the composite action relying upon the surface friction enhanced by the expanded metal meshes tacked welded on the plate, while Unit BS was designed with the composite action relying upon the bearing stresses of concrete and stud shear connectors, where more densely distributed stud shear connectors were provided near the beam-wall joints. Because of the limited loading capacity of the test setup $(500 \mathrm{kN})$, only very thin steel plates ( $3 \mathrm{~mm}$ thick) were used for the short coupling beams. This rendered the use of normal shear studs for Unit BS impossible, as the shank diameter of stud shear connectors had to be close to the plate thickness (i.e. close to $3 \mathrm{~mm}$ in this case) while the smallest available shear stud diameter in the industry is $13 \mathrm{~mm}$. Holes were thus drilled on the plate of Unit BS to accommodate grade 8.8 bolts of nominal diameter $5 \mathrm{~mm}$ for simulating the shear studs. The details of Units SF and BS are described in Figure 2.

Each of the coupling beams was incorporated with a large wall panel at each end for studying the beam-wall interactions. Two base beams were attached at the bottom and the top ends of each $90^{\circ}$-rotated specimen, so that the specimen could be fixed onto the loading frame by screwing bolts into the embedded anchors in the base beams. Loading 
was then applied from a $500 \mathrm{kN}$ actuator to the top end of each specimen through a rigid arm with the line of action passing through the beam center (Figure 3).

Table 1 shows the material properties for all the PRC coupling beams.

\section{Loading History and Instrumentation}

Reversed cyclic loading was first applied to each specimen in a load-controlled cycle up to $75 \%$ of the theoretical ultimate shear capacity $\left(V_{u}^{*}\right)$ to obtain the nominal yield rotation $\left(\theta_{y n}\right)$ at nominal ductility factor $\left(\mu_{n}\right)$ of 1 by the $4 / 3$ rule. Here the beam rotation $(\theta)$ was defined as the differential displacement between the two beam ends in the loading direction divided by the clear span $(l)$. The specimen was then displaced to $\mu_{n}= \pm 1$ for one cycle, and to each successive nominal ductility level for two cycles (Figure 4). The test was terminated when the peak load reached in the first cycle of a nominal ductility level fell below the lesser of $0.8 V_{u}^{*}$ and $80 \%$ of the maximum measured shear $\left(V_{\max }\right)$. The specimen would then be considered to have failed.

The specimens were instrumented with linear variable displacement transducers (LVDTs) to capture the beam deflection profile and the curvatures of beam sections (Figure 5). Strain gauges were attached on the steel plates, the longitudinal bars, the stirrups and some shear studs to investigate the internal load distributions.

\section{RESULTS AND DISCUSSIONS}

\section{Summary of Experimental Findings}

Table 2 summarizes the experimental results. While Units CB and CW reached a value of $V_{\max }$ about $20 \%$ above $V_{u}^{*}$, Unit CF developed a maximum capacity of almost 1.4 times the theoretical capacity. This reveals the highest degree of composite action in Unit CF. A maximum shear stress $\left(v_{\max }\right)$ of $8.58 \mathrm{MPa}$ could be reached in Unit $\mathrm{CF}$, which 
was far above the limit of 5MPa given in BS8110 [17] for conventional RC beams. It has to be noted that the design shear capacity of the $\mathrm{RC}$ was about $2.5 \mathrm{MPa}$.

The performance of short coupling beams was less promising probably due to the relatively smaller plate thickness/beam width ratio and thus a smaller contribution from the plates. With a properly anchored thin plate in the RC section, Unit BS could reach a maximum shear stress of $6.4 \mathrm{MPa}$, which was the same as the theoretical value. Unit SF, however, could only develop $75 \%$ of its theoretical capacity. This shows that the plate was insufficiently anchored and the plate/RC composite action could not be fully developed.

\section{Failure Patterns}

The crack patterns of the specimens after the tests are captured in Figure 6 . The failure modes of the medium-length and the short PRC coupling beams were flexuralshear and shear respectively. As the medium-length beams failed in a more ductile mode than the short beams, the former could dissipate more energy through inelastic deformations.

It is apparent in Figure 6 that plastic hinges were formed in all the three mediumlength coupling beams under large inelastic deformations. The crack patterns suggest that the plastic hinges were located near the beam-wall joints in Units $\mathrm{CB}$ and $\mathrm{CF}$, but were slightly shifted away from the joints in Unit CW. The shift of plastic hinge location in Unit CW might have been due to the comparatively stronger steel plate fixation in the walls with the absence of shear studs in the beam span.

Bond-slip cracks were also formed along the longitudinal reinforcement of these three specimens in the post-elastic stage, but the bond stress that could be developed was much larger than the theoretical value. This shows that although the large bar diameter 
(about $1 / 10$ of the beam width) may not significantly affect the beam capacity, it may adversely affect the inelastic behavior of the beam under large imposed deformations.

No sign of bond-slip could be observed in the short coupling beams, as the behavior of these beams are normally governed by shear, and sliding cracking is the major problem in short RC coupling beams sufficiently reinforced in shear. The use of longitudinal reinforcement with a smaller diameter (1/12 of the beam width) might also have helped in preventing the bond-slip.

Both diagonal cracks and sliding cracks at the beam-wall joints could be observed in the short coupling beams, but the latter became dominant as the loading progressed. The cracks in Unit BS were more severe than those in Unit SF before failure. This indicates that the plate in the former took up a more important role in the shear transfer across the beam-wall joints. Thus the shear transfer was less affected by the loss of aggregate interlock due to sliding cracking.

\section{Hysteretic Responses}

Figure 7 shows the load-chord rotation $(V-\theta)$ curves of the specimens. The responses of Units $\mathrm{CF}$ and $\mathrm{CW}$ are almost similar, but that of Unit $\mathrm{CB}$ is much less desirable as pinching could be observed in this unit. While Units CF and CW could respond immediately to the change in loading direction, Unit CB deformed considerably under a small loading upon load reversal.

The difference in the above behavior could have been due to the absence of shear studs in the wall regions of Unit CB causing anchorage bond-slip of the plate. As a result, shear transfer through the plate could not be activated. Therefore, the plate of a PRC coupling beam should be firmly anchored in the wall piers to ensure a ductile beam performance. The drastically deviating responses between Units SF and BS gave further corroboration to this account. It could also be observed that insufficient anchorage of the 
embedded steel plate in the wall regions would even result in prevention of full-strength development in short PRC coupling beams.

Though fully provided with shear studs in the wall and the beam regions, Unit BS, unlike its medium-length counterpart Unit CF, still underwent apparent pinching upon reversal of loading direction. The pinching problem could have been partially due to slips between the bolts and the plate (due to the presence of hole clearance) that reduced the degree of plate/RC interaction. Also, because of the densely distributed holes in the highly stressed areas in the walls near the beam-wall joints, the plate strength was weakened and the plate started to crack in the most critical region as loading progressed. The gradual cracking of the plate reduced the effectiveness of shear transfer across the beam-wall joints, thus causing pinching and failure of the beam eventually.

Although the shear studs in the beam span seem to have little effect on the beam performance, the larger capacity developed in Unit CF compared with Units CB and CW suggests that these shear studs would help to develop a higher degree of composite action, i.e. a better utilization of the components, in the coupling beam.

The coupling beams all underwent strength degradations in the repeated cycles mainly due to concrete cracking in the first cycle that reduced the load carrying capacity of the concrete. The medium-length PRC coupling beams were able to retain a higher percentage of strength than the short coupling beams when a load cycle was repeated. This was because the reinforced concrete took up a smaller share of the load resistance in the former group than in the latter group. Thus if a thicker plate had been provided in Unit BS to take up a larger share of load resistance, the strength degradation problem could have been much alleviated. The problem of serious strength degradation upon repetition of a load cycle in Unit SF, however, was due to poor plate/RC interaction. As the plate had not been effectively utilized, concrete cracking resulted in a considerable loss of the load carrying capacity of the beam. 


\section{Energy Dissipation}

In order to quantify the seismic performances of the PRC coupling beams, the amounts of cumulative energy dissipated by the specimens are compared. To facilitate comparison, in Figure 8 the cumulative energy dissipated $\left(W_{d, \text { cum }}\right)$ by each specimen is normalized by the energy dissipated in the positive nominal yield cycle $\left(W_{d, y n}\right)$. This eliminates the pre-existing differences in section properties like section dimensions, steel reinforcement and plate contents, concrete strength, etc. While $W_{d, c u m}$ is proportional to the total area enclosed by the load-rotation curve up to the corresponding stage, $W_{d, y n}$ is proportional to the area enclosed by the load-rotation curve in the cycle of $\mu_{n}=1$.

Unit CF demonstrated the best energy dissipation ability among the five specimens, while Unit SF could not dissipate much energy. The poor performance of Unit SF was due to the ineffective plate/RC shear transfer that resulted in little contribution of the embedded steel plate in energy dissipation.

Despite the difference in span/depth ratio and shear stud arrangement, the performances of Units $\mathrm{CB}$ and $\mathrm{BS}$ were almost similar with regard to the energy dissipation ability. Although more densely distributed stud shear connectors were provided near the beam-wall joints to further enhance the plate/RC composite action, Unit BS could not perform as desirably as Unit CF. The reason was probably due to the presence of densely distributed holes that weakened the plate as discussed in the above section. However, with shear studs welded on the plate, rather than having bolts screwed onto the plate, in a real PRC coupling beam, the energy dissipation ability should be better than that of Unit BS.

\section{Strain Distributions}

The strain distributions along the top reinforcement and the top fibers of the plates at the positive cycle peaks are shown in Figures 9 and 10 respectively. In the 
medium-length PRC coupling beams, the strain profiles of the plates were more or less anti-symmetrical about the mid-span of the beams, while the points of zero strain continuously shifted towards the compression ends in the longitudinal reinforcement. The short PRC coupling beams, on the other hand, had the points of contra-flexure in the reinforcement and the plates remained near the mid-span, but the strain profiles were not anti-symmetrical about the mid-span of the beams. Rather, the tensile strains in these beams were consistently far higher than the compressive strains. This indicates that the concrete could have been the major component taking up compression in the beams However, the fact that both the top reinforcement and the plate top fiber were mainly under tension throughout the beam span also suggests that the major shear resistance mechanism could have shifted from the truss action to the arch action. Under the arch action, a diagonal strut joining the compression corners presses against the wall piers, inducing tensile forces along the top and bottom steel as its horizontal component pushes the wall piers apart [10].

In the short PRC coupling beam series, the shape of the reinforcement strain profile is almost similar to that of the plate strain profile in each beam, unlike the case of drastic difference between the reinforcement and the plate strain profiles in each medium-length PRC coupling beam. This suggests that the plate and the reinforcement responses were closer to each other in the former series, as bond-slips of the longitudinal reinforcement in the latter caused the reinforcement unable to deform together with the beams under inelastic deformations.

The plate strains were generally lower in Unit SF than in Unit BS. This shows that the smaller strength of Unit SF was mainly due to the smaller resistance provided by the embedded plate. 


\section{THEORETICAL MODEL FOR PLATE ANCHORAGE DESIGN}

As demonstrated earlier by the experimental results, a proper plate anchorage design is of vital importance to the ductile performance of a PRC coupling beam. When the design of PRC coupling beams was first introduced, a simple model assuming uniform bearing stress distributions in the wall embedment region was employed and the plate was designed to transfer the whole design shear and the design end moment of the plate from the coupling beam to the wall piers [16]. Shear studs were then arranged at uniform spacing on the plate in the wall regions, which was the case in Units CW and CF.

It should be noted that the simple model assuming uniform bearing stress distribution, which includes an abrupt change of bearing force direction, is only valid for rigid-plastic materials. However, reinforced concrete only possesses limited ductility. This leads to non-uniform stress distribution in the stress blocks. It is therefore more rational to assume a bearing stress distribution that takes into account the gradual change of bearing stress.

Based on the experimental observations on the plate anchors of Unit CF with evenly distributed shear studs, the design model in Figure 11 with a simplified bearing stress distribution at ultimate limit state is proposed. The lengths of the rectangular stress blocks near the end of the anchor and the beam-wall joint are respectively $\alpha L_{e}$ and $\beta L_{e}$, where $\alpha$ and $\beta$ are dimensionless variables, and $L_{e}$ is the total anchorage length of the embedded steel plate. The bearing stresses (in force per unit length) of the two rectangular stress blocks are $\gamma w$ and $w$ respectively, where $\gamma(\leq 1)$ is a constant to be determined from experimental results.

In order to find out a suitable combination of $\alpha$ and $\beta$ values to achieve moment and shear equilibrium, the value of either $\alpha$ or $\beta$ should be fixed first. It can be observed that the value of $\alpha(\leq 1)$ increases as the plate moment-to-shear ratio at the beam-wall 
joint $\left(M_{p} / V_{p}\right)$ varies from 0 (i.e. under pure shear) to infinity (i.e. under pure bending). Suppose $\alpha$ varies from $b(\geq 0)$ to $a+b(\leq 1), \alpha$ can be determined from the following equation:

$$
\alpha=a[\lambda(2 \sqrt{2}+2)-\sqrt{2}]^{n}+b, 0 \leq \alpha \leq 1
$$

where $a, b$ and $n$ are some constants to be determined from the experimental results, while the dimensionless variable $\lambda$ is governed by the following equation that relates the lengths of the rectangular stress blocks in a reference case shown in Figure 12:

$$
\lambda=\frac{\alpha_{0}}{\alpha_{0}+\beta_{0}}=\frac{\sqrt{\frac{M_{p}}{w}+\frac{V_{p}^{2}}{2 w^{2}}}}{2 \sqrt{\frac{M_{p}}{w}+\frac{V_{p}^{2}}{2 w^{2}}}+\frac{V_{p}}{w}}, \frac{1}{2+\sqrt{2}} \leq \lambda \leq \frac{1}{2}
$$

Considering shear and moment equilibriums:

$$
\begin{aligned}
& V_{p}=\frac{\beta L_{e} w}{2}(1+\gamma)+\frac{L_{e} w}{2}(1-\alpha-\gamma-\alpha \gamma) \\
& M_{p}=\left[\frac{\gamma L^{\prime}}{2}\left(\frac{\gamma}{1+\gamma}\right)\left(\frac{(3+2 \gamma) L^{\prime}}{3(1+\gamma)}+\beta L_{e}\right)+\alpha \gamma L_{e}\left(L_{e}-\frac{\alpha L_{e}}{2}\right)-\frac{\beta^{2} L_{e}{ }^{2}}{2}-\frac{L^{\prime}}{2(1+\gamma)}\left(\frac{L^{\prime}}{3(1+\gamma)}+\beta L_{e}\right)\right] w
\end{aligned}
$$

where $L^{\prime}=(1-\alpha-\beta) L_{e}$

Rearranging the above equations,

$$
L_{e}=\frac{A V_{p}+\sqrt{A^{2} V_{p}^{2}+4 B\left(4 V_{p}^{2}+6 M_{p} w(1+\gamma)\right)}}{2 w B}
$$

where $A=2 \alpha \gamma+6 \gamma+2 \alpha-2$ and $B=-1-2 \alpha-\alpha^{2}+3 \gamma+2 \alpha \gamma-2 \alpha^{2} \gamma-\alpha^{2} \gamma^{2}$

Note that according to BS5950 [18], when designing conventional composite beams with RC slabs and structural steel beams interconnected by shear studs, the shear stud strength is considered to be mobilized by $80 \%$ and $60 \%$ under positive and negative moments respectively taking into account the different interface slips available.

Following this principle, a maximum shear stud mobilization of $60 \%$ only is considered in the zone within a length of $\beta L_{e}$ from the beam-wall joint. Therefore, $w$ is related with 
the number of shear studs in a vertical section $\left(n_{w}\right)$, horizontal spacing of shear studs $\left(s_{w}\right)$, characteristic resistance of shear studs $\left(Q_{k}\right)$, characteristic concrete cube strength $\left(f_{c u}\right)$ and plate thickness $(t)$ by the following equation:

$$
w=\frac{0.6 n_{w} Q_{k}}{s_{w}}+0.8 f_{c u} t
$$

By providing evenly distributed shear studs throughout the wall anchorage region of the steel plate, smaller degrees of maximum shear stud mobilization are automatically applied in the other three zones.

The revised model has been applied to determine the bearing stress distribution in the plate anchor of Unit CF at the stage when the specimen was almost loaded to its peak load. As strain gauges have been installed on the surface of the embedded steel plate at the wall anchor, shear force variation as well as the bearing stress distribution could be calculated from the experimental results. The distribution is compared with those obtained from the original design model [16] as well as the revised model in Figure 13. The following set of values for the constants was adopted when applying the revised model:

$$
a=0.25, b=0.05, n=2 \text { and } \gamma=0.65
$$

Figure 13 shows that the original design model has underestimated the maximum bearing stress on the plate near the beam-wall joint, while the revised model can give a much better estimation of the bearing stress distribution. Furthermore, the new model also leads to a better and closer estimation of the plate moment and shear distributions at the wall anchors. Refinement of the proposed model will be carried out for recommending an appropriate range of values for each constant. 


\section{CONCLUSIONS}

The experimental results of five PRC coupling beams of two different span-depth ratios subjected to reversed cyclic loading have been reported in this paper. The following conclusions are drawn:

1. Shear studs provided in the beam span of a PRC coupling beam can slightly enhance the plate/RC interaction, thus giving a slightly higher beam capacity. They also contribute to the better utilization of the components in a PRC coupling beam in the inelastic stage.

2. The absence of shear studs in the beam span will result in a relatively stronger fixation of the plate in the wall regions than in the beam span, and the plastic hinges will shift slightly away from the beam-wall interfaces into the beam span.

3. The absence of shear studs in the wall regions will cause anchorage bond-slip of the plate, which inactivates the shear transfer across the beam-wall joints through the plate. Poor anchorage of the plate in the wall regions can even hinder the development of full strength in a short PRC coupling beam.

4. To ensure desirable performance of a PRC coupling beam, especially in resisting inelastic deformations, the embedded steel plate has to be effectively anchored in the wall piers. This can be achieved by providing shear studs on the plate surfaces in the wall regions.

5. When the plate in a PRC coupling beam takes up a larger share of load resistance, the beam will be able to retain a higher percentage of its strength upon repetition of a load cycle. A more ductile beam performance can also be achieved as the plate assumes a more important role in shear transfer across the beam-wall joints, which is less affected by the loss of aggregate interlock due to sliding cracking.

6. The poor performance of Unit SF was due to ineffective plate/RC shear transfer that resulted in little contribution of the steel plate in load resistance and energy 
dissipation. Therefore, the use of expanded metal meshes may not be an effective way in enhancing the plate/RC composite action of a PRC coupling beam.

Based on the experimental observations, the design model of simplified bearing stress distribution in the plate anchorage is revised. An example comparing the experimental anchorage bearing stress distribution with the theoretical distributions derived from the original and the revised models has shown a much improved prediction of the anchorage behavior by the revised model. 


\section{ACKNOWLEDGEMENTS}

The research described here has been fully supported by the Research Grants Council of Hong Kong SAR (Project No. HKU7129/03). Technical supports for the experimental work provided by the laboratory staff of the Department of Civil Engineering, The University of Hong Kong, are gratefully acknowledged. Mr. Tony Earhart is also gratefully thanked for his proofreading of the paper.

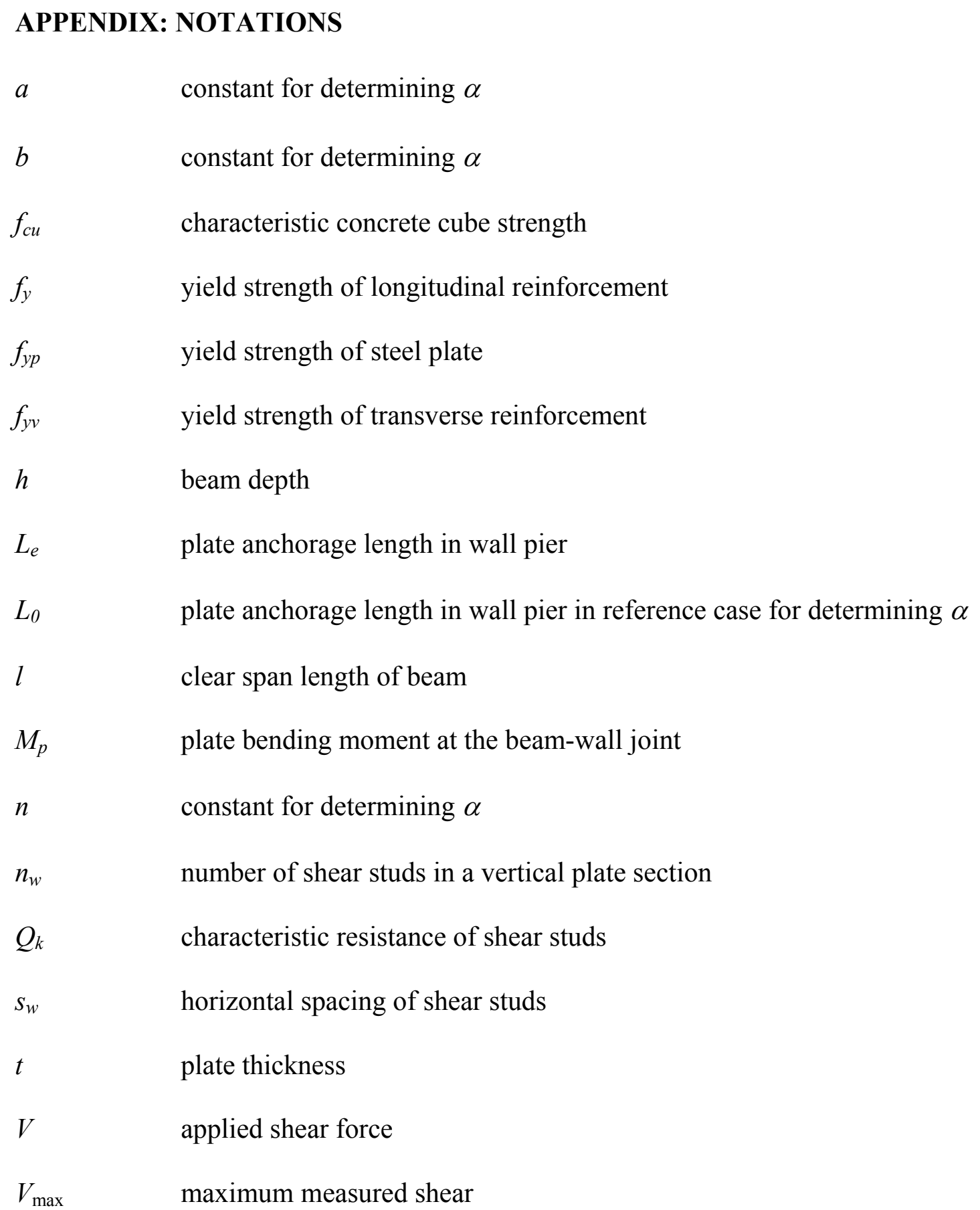




\begin{tabular}{|c|c|}
\hline$V_{p}$ & plate shear at the beam-wall joint \\
\hline$V_{\text {Peak } 1}$ & peak load in the first cycle of a ductility level \\
\hline$V_{\text {Peak } 2}$ & peak load in the repeated cycle of a ductility level \\
\hline$V_{u}^{*}$ & theoretical ultimate shear capacity \\
\hline$v_{\max }$ & maximum shear stress \\
\hline$W_{d, \text { cum }}$ & cumulative energy dissipated \\
\hline$W_{d, y n}$ & energy dissipated in the positive nominal yield cycle \\
\hline$w$ & bearing stress on steel plate \\
\hline$\alpha$ & $\begin{array}{l}\text { ratio of length of rectangular stress block near end of plate anchor to total } \\
\text { plate anchorage length in wall pier }\end{array}$ \\
\hline$\alpha_{0}$ & $\begin{array}{l}\text { ratio of length of rectangular stress block near end of plate anchor to total } \\
\text { plate anchorage length in wall pier in reference case for determining } \alpha\end{array}$ \\
\hline$\beta$ & $\begin{array}{l}\text { ratio of length of rectangular stress block near beam-wall joint to total } \\
\text { plate anchorage length in wall pier }\end{array}$ \\
\hline$\beta_{0}$ & $\begin{array}{l}\text { ratio of length of rectangular stress block near beam-wall joint to total } \\
\text { plate anchorage length in wall pier in reference case for determining } \alpha\end{array}$ \\
\hline$\gamma$ & $\begin{array}{l}\text { ratio of maximum plate bearing stress near end of plate anchor to } \\
\text { maximum plate bearing stress near beam-wall joint }\end{array}$ \\
\hline$\lambda$ & dimensionless variable for determining $\alpha$ \\
\hline$\theta$ & chord rotation of beam \\
\hline$\theta_{\max }$ & maximum chord rotation of beam \\
\hline$\theta_{y n}$ & nominal yield chord rotation of beam \\
\hline$\mu_{n}$ & nominal ductility factor \\
\hline$\mu_{\max n}$ & maximum nominal ductility factor \\
\hline
\end{tabular}




\section{REFERENCES}

1. Paulay T. 1971. Coupling Beams of Reinforced Concrete Shear Walls. Proceedings of ASCE 97(ST3): $843-862$.

2. Wang W., Fu X. \& Ni Y. 1992. Behavior of Ceramite Concrete Coupling Beams in Shear Wall Structure under Reversed Cyclic Loading (in Chinese). Journal of Building Structures 13(1): $71-78$

3. Paulay T. \& Binney J.R. 1974. Diagonally Reinforced Coupling Beams of Shear Walls. ACI Special Publication SP42: 579 - 598.

4. Tegos I.A. \& Penelis G.G. 1988. Seismic Resistance of Short Columns and Coupling Beams Reinforced with Inclined Bars. ACI Structural Journal 85(1): 82 - 88.

5. Subedi N.K. 1989. Reinforced Concrete Beams with Plate Reinforcement for Shear. Proceedings of the Institution of Civil Engineers Part 1 - Design \& Construction. 87: $377-399$.

6. Harries K.A., Mitchell D., Cook W.D. \& Redwood R.G. 1993. Seismic Response of Steel Beams Coupling Concrete Walls. Journal of Structural Engineering, ASCE 119(12): $3611-3629$.

7. Gong B. and Shahrooz B.M. 2001. Concrete-steel Composite Coupling Beams I: Component Testing. Journal of Structural Engineering, ASCE, 127(6): 625 - 631.

8. Teng J.G., Chen J.F. \& Lee Y.C. 1999. Concrete-filled Steel Tubes as Coupling Beams for RC Shear Walls. Proceedings, Second International Conference on Advances in Steel Structures 15-17 December 1999, Hong Kong: 391 - 399.

9. Lam W.Y., Su R.K.L. \& Pam H.J. 2001. Embedded Steel Composite Coupling Beams - a New Proposal. Proceedings of International Conference on Construction 19-21 June 2001, Hong Kong, 2: 93 - 104.

10. Paulay T. 1971. Coupling Beams of Reinforced Concrete Shear Walls. Proceedings of ASCE 97(ST3): $843-862$. 
11. Pam H.J., Su R.K.L., Lam W.Y., Li J., Au F.T.K., Kwan A.K.H. and Lee P.K.K. 2002. Designing Coupling Beams and Joints in Concrete Buildings for Improved Earthquake Resistance. Proceeding, One Day Seminar on Recent Developments in Earthquake Engineering, 17 May 2002, HKIE and IstructE: 91-105.

12. Lam W.Y., Su R.K.L. \& Pam H.J. 2002. The Performance and Design of Embedded Steel Composite Coupling Beams. Proceeding of Structural Engineers World Congress 9-12 October 2002, Yokohama, Japan (in CD Rom).

13. Lam W.Y., Su R.K.L. and Pam H.J. 2003. Strength and Ductility of Embedded steel Composite Coupling Beams. International Journal of Advances in Structural Engineering, 6(1): 23-35.

14. Lam W.Y., Su R.K.L. and Pam H.J. 2004. The Effects of Shear Studs on Platereinforced Composite Coupling Beams. Proceedings of The Second International Conference on Structural Engineering, Mechanics and Computation, 4-7 July, Cape Town, South Africa (in CD Rom).

15. Lam W.Y., Su R.K.L. and Pam H.J. 2004. Seismic Performance of Plate Reinforced Composite Coupling Beams. Proceeding of $13^{\text {th }}$ World Congress on Earthquake Engineering, 1-6 August 2004, Vancouver, Canada (in CD Rom).

16. Lam W.Y., Su R.K.L. and Pam H.J. 2004. Experimental Study on Embedded Steel Plate Composite Coupling Beams. Journal of Structural Engineering, ASCE (in press).

17. BSI 1985. BS8110, Part 1, Code of Practice for Design and Construction. British Standards Institution 1985, London.

18. BSI 1990. BS5950, Structural Use of Steelwork in Building, Part 3: Design in Composite Construction, Section 3.1: Code of Practice for Design of Simple and Continuous Composite Beams. British Standards Institution 1990, London. 
Figure 1. Reinforcement Cages of PRC Coupling Beams on Site

Figure 2. Specimen Geometries and Reinforcement Details

Figure 3. Test Set-up and Loading Application

Figure 4. Loading History

Figure 5. LVDT Arrangement

Figure 6. Failure Patterns of Test Specimens

Figure 7. Load-chord rotation Curves

Figure 8. Normalized Cumulative Energy Dissipated

Figure 9. Strain Profiles of Top Flexural Reinforcement

Figure 10. Strain Profiles along Top Fibers of Steel Plates

Figure 11. Proposed Model for Plate Anchorage Design

Figure 12. Reference Bearing Stress Distribution in Wall Embedment Region for

Determining $\alpha$

Figure 13. Comparison between Experimental and Theoretical Bearing Stress

Distributions of Plate Anchor 
Figure 1
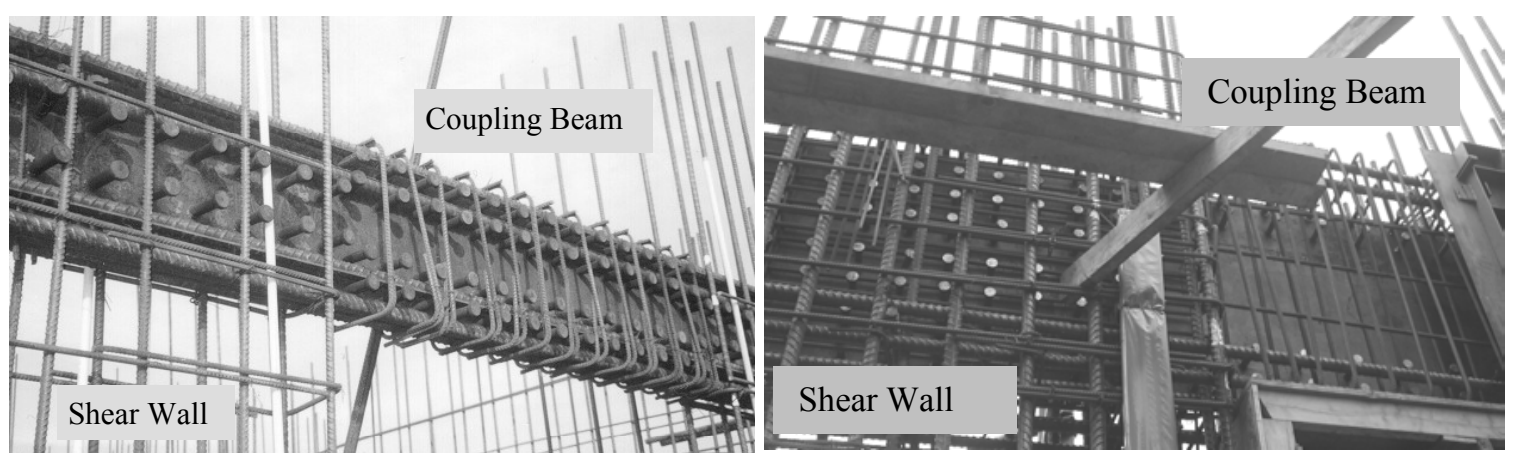

Figure 2
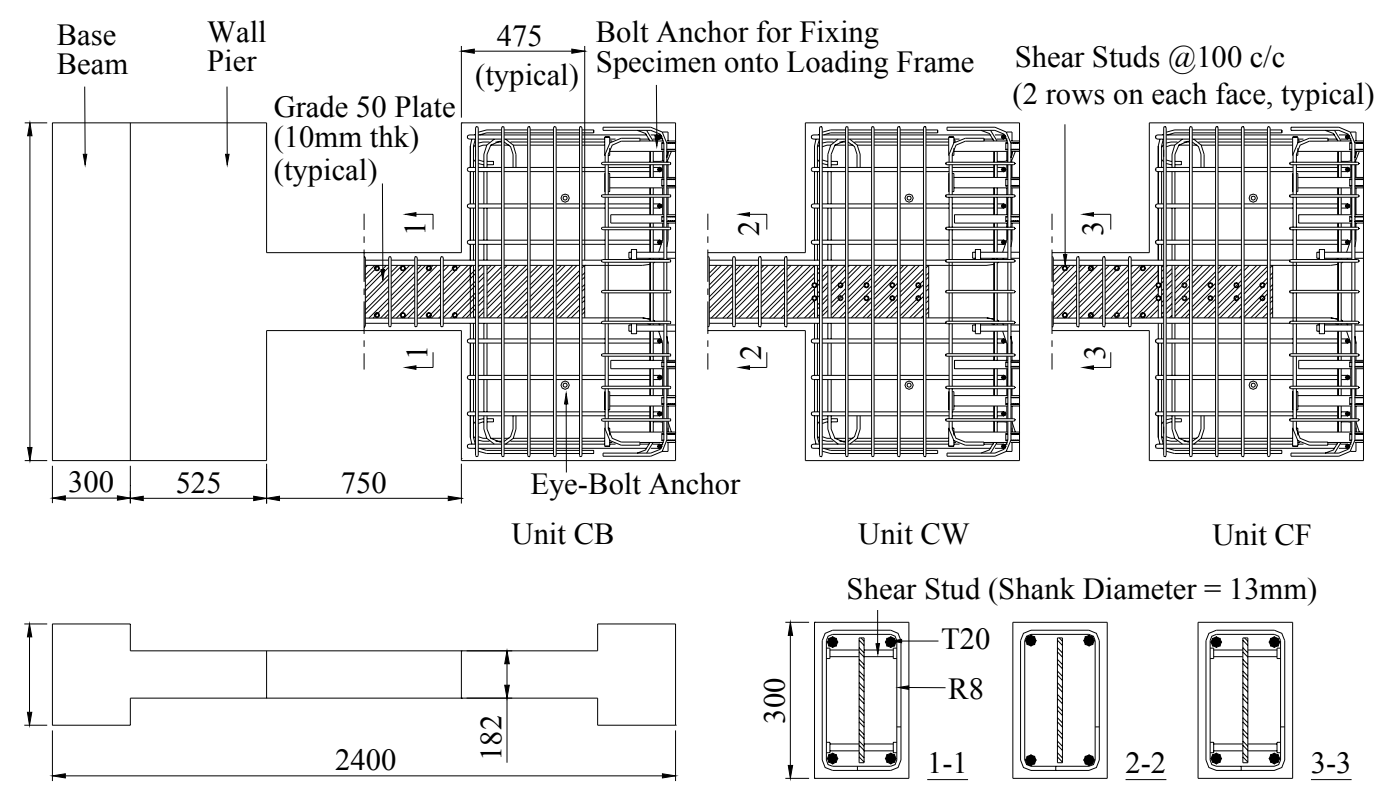

Shear Stud $($ Shank Diameter $=13 \mathrm{~mm})$

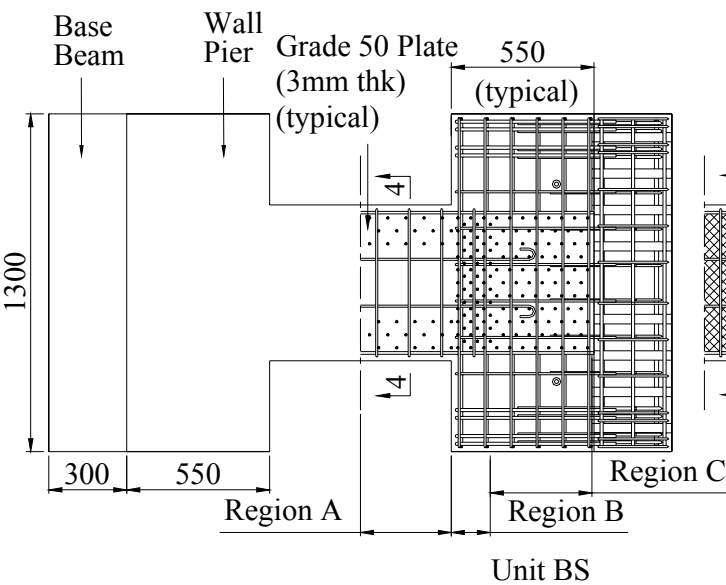

Expanded Metal Mesh (welded on plate surface)
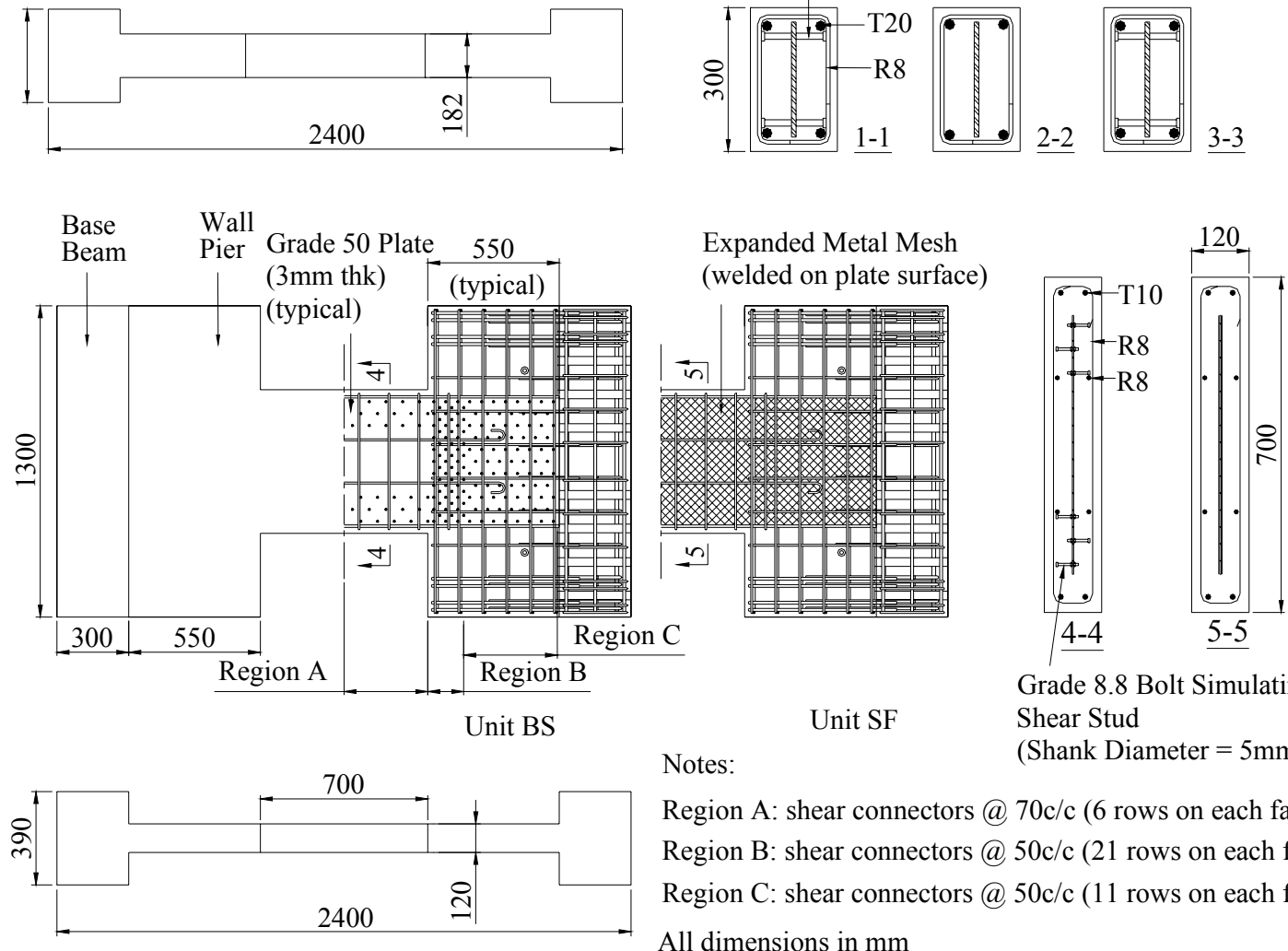

Notes:

Grade 8.8 Bolt Simulating Shear Stud

$($ Shank Diameter $=5 \mathrm{~mm})$

Region A: shear connectors @ $70 \mathrm{c} / \mathrm{c}$ (6 rows on each face) Region B: shear connectors @ 50c/c (21 rows on each face) Region C: shear connectors @ 50c/c (11 rows on each face) All dimensions in $\mathrm{mm}$ 
Figure 3

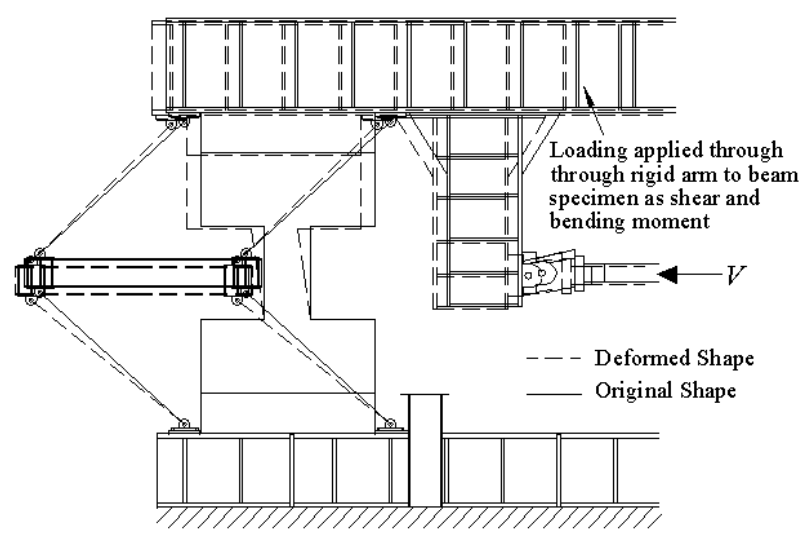

Figure 4

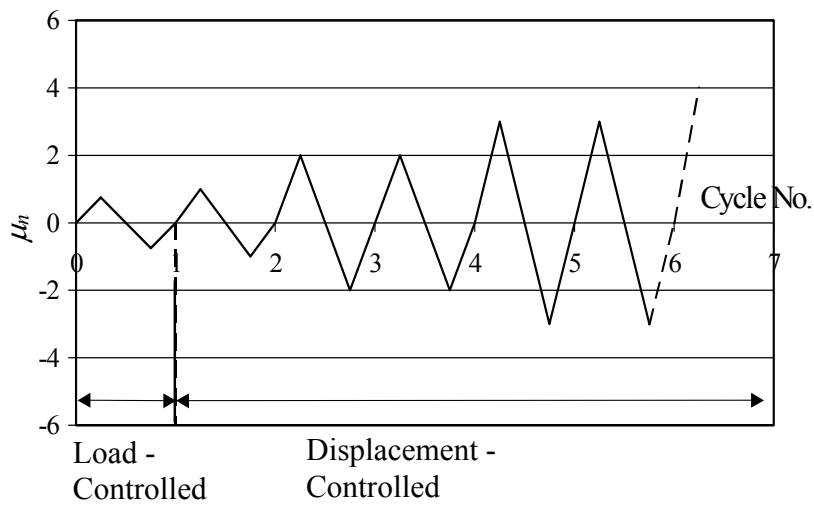

Figure 5

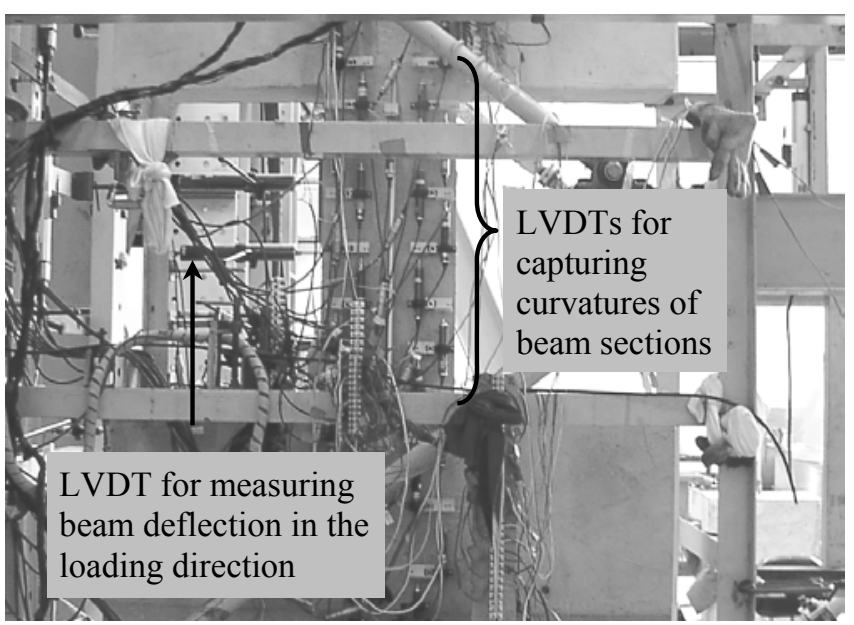


Figure 6

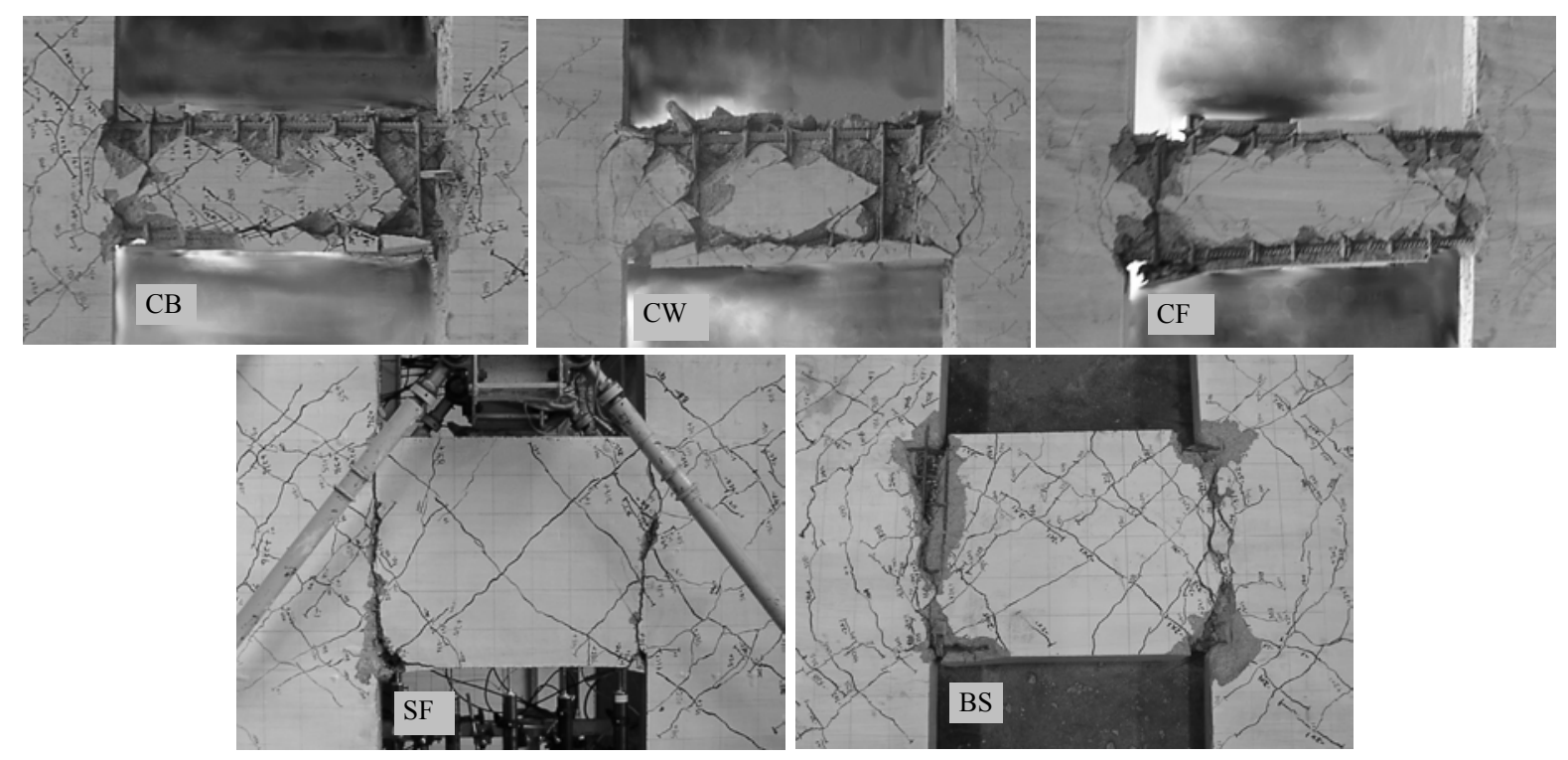

Figure 7
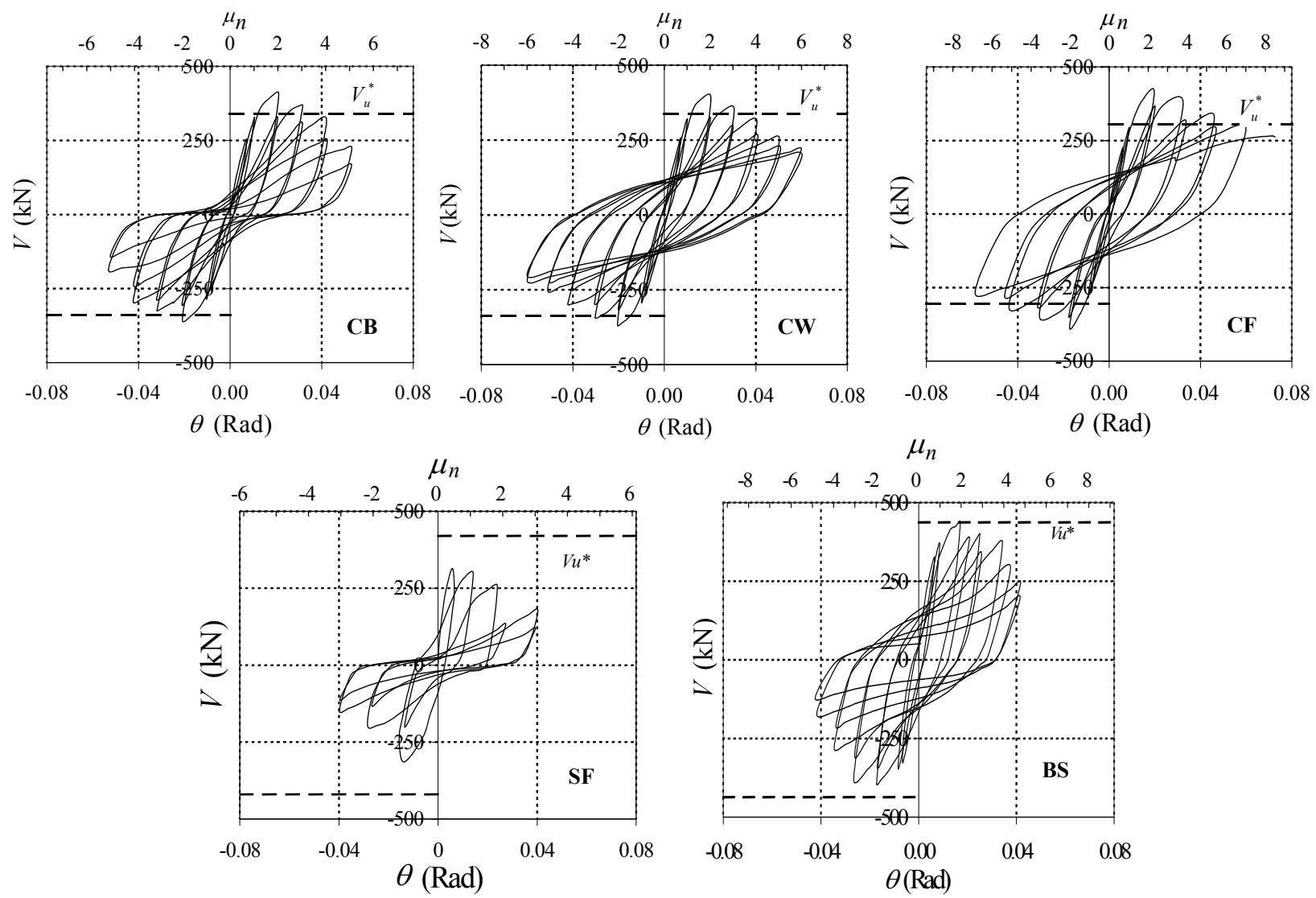
Figure 8

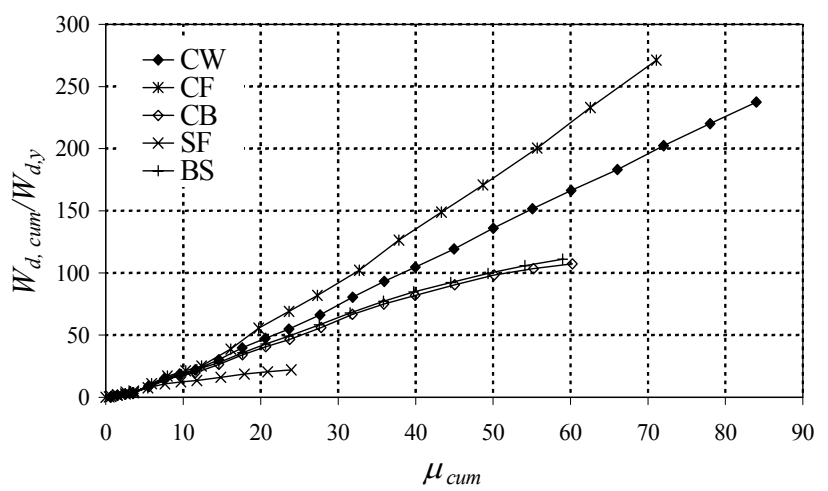

Figure 9
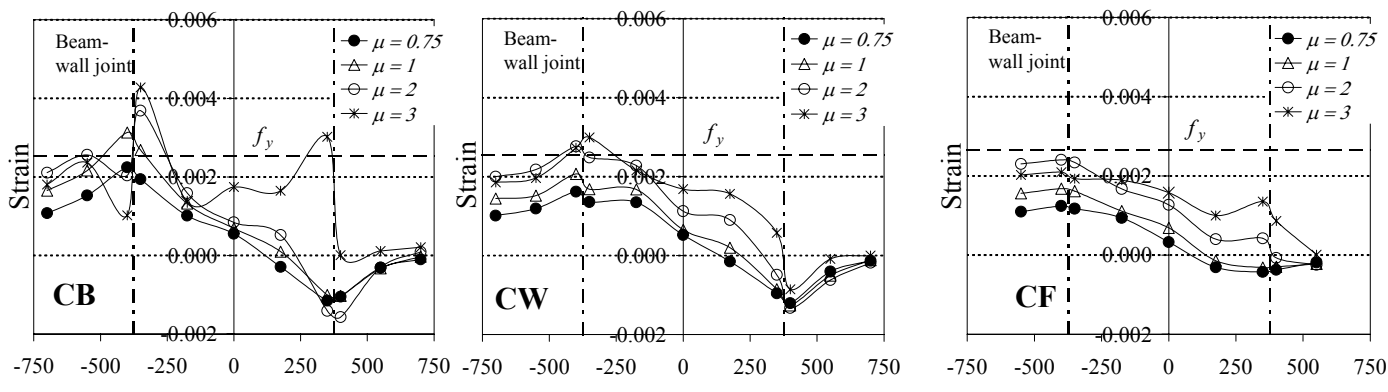

Distance from Beam Center (mm)

Distance from Beam Center (mm)

Distance from Beam Center (mm)
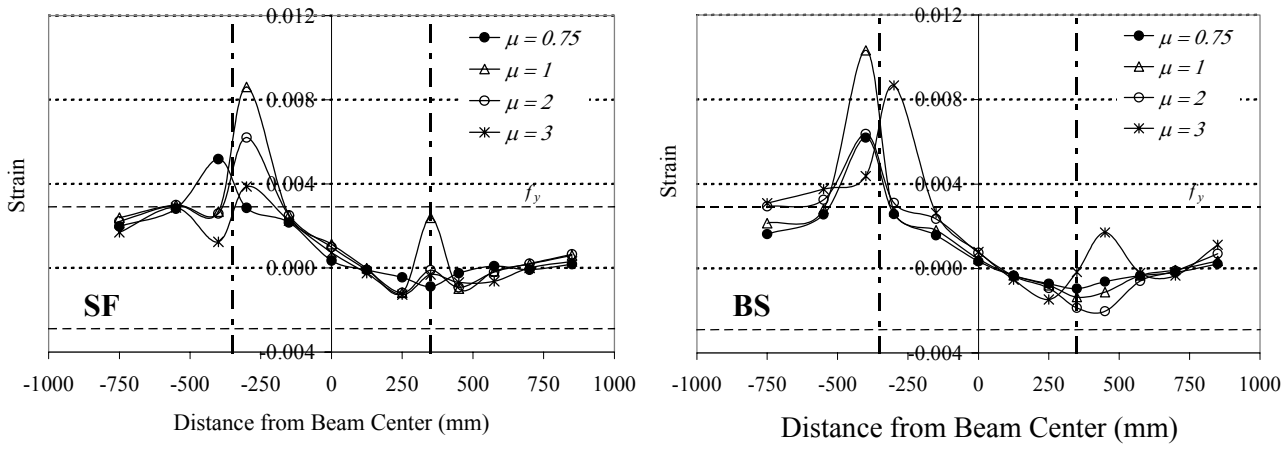
Figure 10
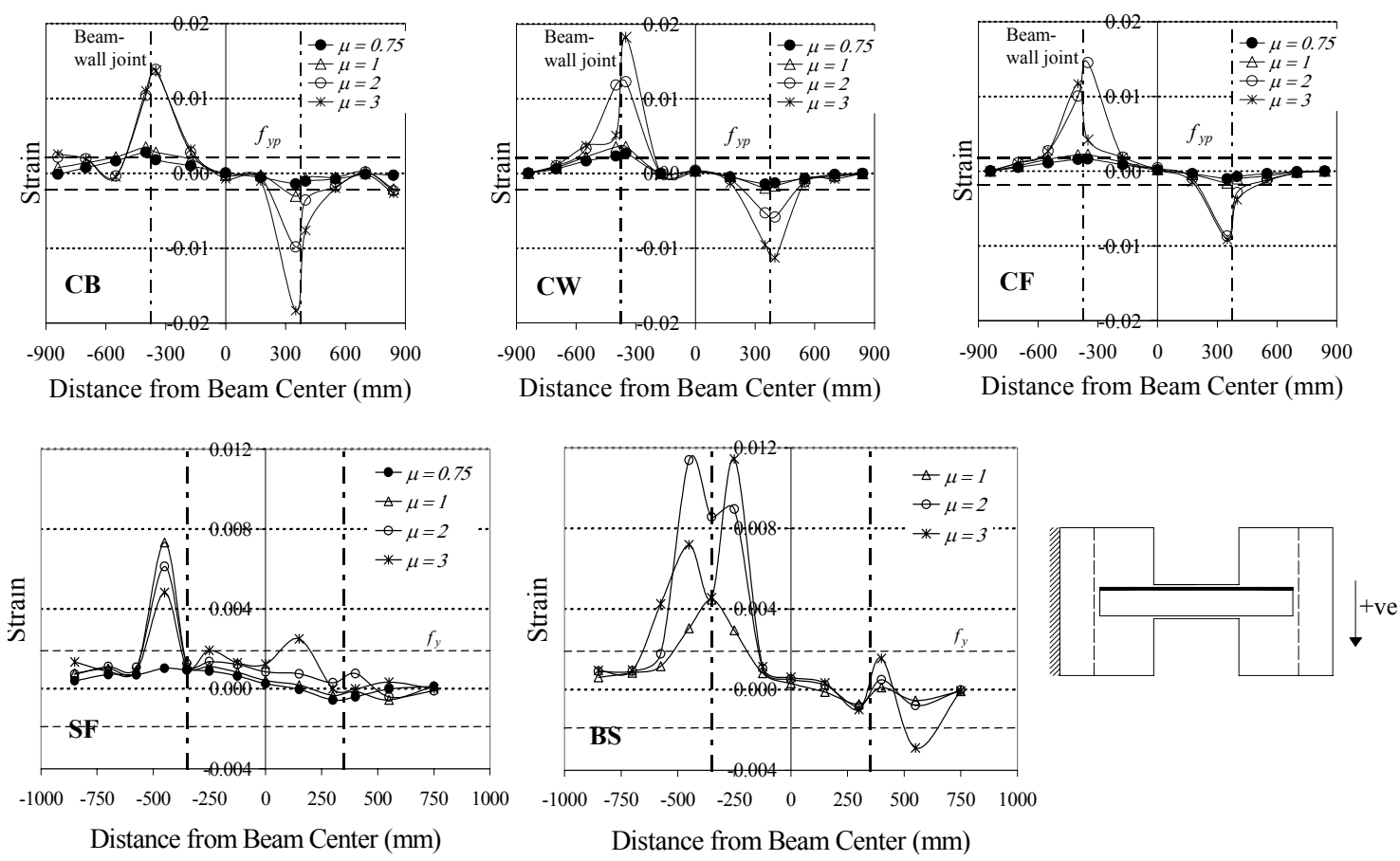

Figure 11

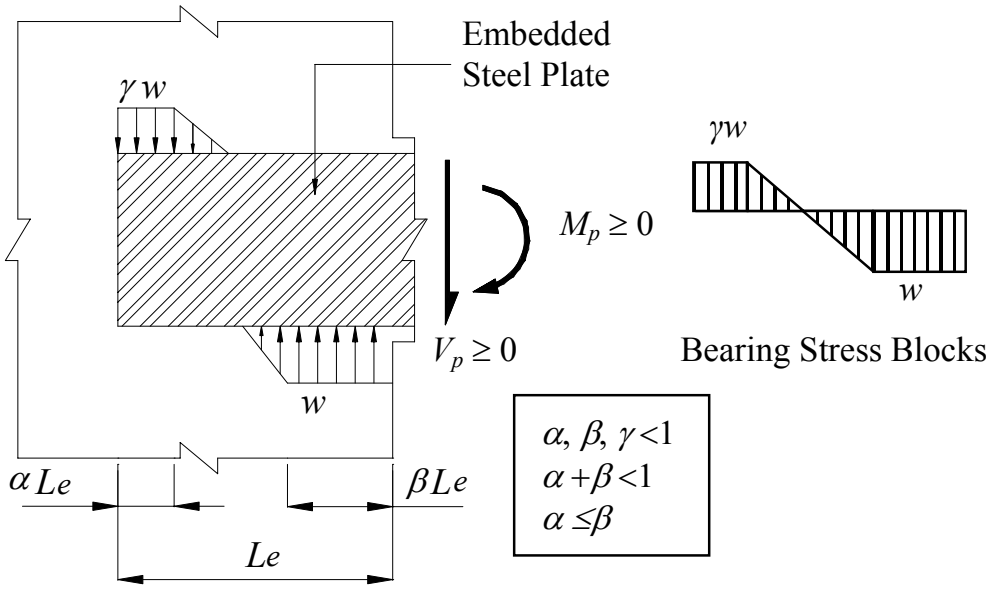


Figure 12

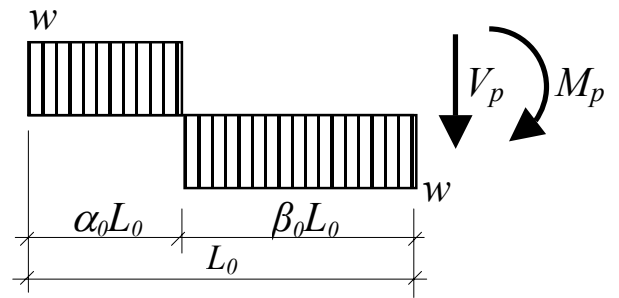

Figure 13

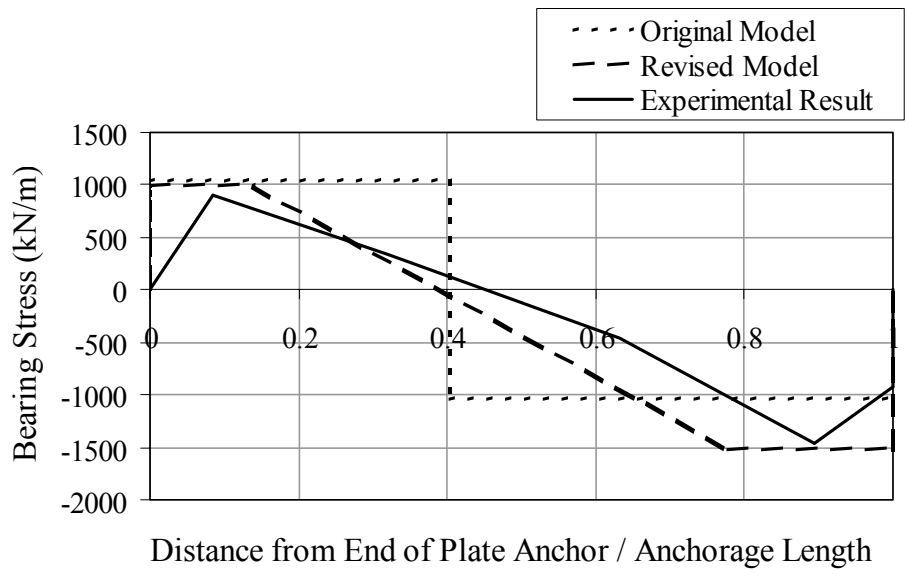


Table 1. Material Properties

\begin{tabular}{cccccccc}
\hline \multirow{2}{*}{ Specimen } & $f_{y}$ & $f_{u}$ & $f_{y v}$ & $f_{u v}$ & $f_{y p}$ & $f_{y p}$ & $f_{c u}$ \\
\cline { 2 - 8 } & $\mathrm{MPa}$ & $\mathrm{MPa}$ & $\mathrm{MPa}$ & $\mathrm{MPa}$ & $\mathrm{MPa}$ & $\mathrm{MPa}$ & $\mathrm{MPa}$ \\
\hline Unit CB & 514 & 646 & 392 & 541 & 435 & 580 & 50.8 \\
\hline Unit CW & 514 & 646 & 392 & 541 & 435 & 580 & 53.7 \\
\hline Unit CF & 523 & $\mathrm{~N} / \mathrm{A}$ & 367 & 468 & 370 & 509 & 51.9 \\
\hline Unit SF & 541 & 652 & 354 & 488 & 405 & 498 & 48.1 \\
\hline Unit BS & 541 & 652 & 354 & 488 & 405 & 498 & 50.0 \\
\hline
\end{tabular}

Notes: $f_{y}=$ yield strength of longitudinal reinforcement

$f_{u}=$ ultimate strength of longitudinal reinforcement

$f_{y v}=$ yield strength of transverse reinforcement

$f_{u v}=$ ultimate strength of longitudinal reinforcement

$f_{y p}=$ yield strength of steel plate

$f_{u p}=$ ultimate strength of steel plate

$f_{c u}=$ concrete cube strength 
Table 2. Summary of Experimental Results

\begin{tabular}{ccccccc}
\hline Specimen & $V_{\max }$ & $v_{\max }$ & $V_{\max } / V_{u}^{*}$ & $\theta_{y n}$ & $\theta_{\max }$ & $\mu_{\max n}$ \\
\cline { 2 - 7 } & $\mathrm{kN}$ & $\mathrm{MPa}$ & & $\mathrm{Rad}$ & $\mathrm{Rad}$ & \\
\hline Unit CB & 408 & 8.40 & 1.20 & 0.0104 & 0.0531 & 5.1 \\
\hline Unit CW & 397 & 8.17 & 1.18 & 0.0100 & 0.0599 & 6.0 \\
\hline Unit CF & 417 & 8.58 & 1.37 & 0.0085 & 0.0785 & 9.2 \\
\hline Unit SF & 315 & 4.61 & 0.75 & 0.0131 & 0.0403 & 3.1 \\
\hline Unit BS & 438 & 6.40 & 1.00 & 0.0087 & 0.0424 & 4.9 \\
\hline
\end{tabular}

